\title{
Implementing Global Masters into Parallel Condensation
}

\author{
Andreas Pelzer, Birgit Hofferek and Heinrich Voss \\ Section of Mathematics \\ Technical University of Hamburg-Harburg \\ D - 21071 Hamburg \\ Germany \\ voss @ tu-harburg.de http://www.tu-harburg.de/mat/
}

Abstract: The dynamic analysis of structures leads to very large generalized eigenvalue problems. Their number of unknowns can be reduced to a manageable size by condensation methods which can be parallelized by combining it with substructuring. In this note we implement global masters into the parallel condensation process.

Keywords: eigenvalue problem, condensation, global masters,

\section{Condensation with general masters}

In the analysis of the dynamic response of structures using finite element methods very often prohibitively many degrees of freedom are needed to model the behaviour of the system sufficiently accurate. Static condensation is frequently employed to economize the computation of a selected group of eigenvalues and eigenvectors. These methods choose from the degrees of freedom a small number of master variables. Neglecting inertia terms the remaining variables (termed slaves) are eliminated leaving a much smaller problem for the master variables only.

It has frequently been noted in the literature that the quality of the eigenvalue and eigenvector approximations produced by static condensation is satisfactory only for a very small part of the lower end of the spectrum. To improve the approximation properties of condensation Mackens and the 
third author [3] introduced general masters which allow to implement a priori information of the eigenmodes (such as eigenmodes of similar structures considered in reanalysis or prolongations of eigenvector approximations obtained on a coarser grid) into the condensation process.

We consider the general eigenvalue problem

$$
K x=\lambda M x
$$

where $K \in \mathbb{R}^{(n, n)}$ and $M \in \mathbb{R}^{(n, n)}$ are symmetric and positive definite matrices which are usually the stiffness and mass matrix of a finite element model of a structure, respectively. The dimension $n$ is supposed to be very large.

To reduce the number of degrees of freedom we choose linear independent vectors $z_{1}, \ldots, z_{m} \in \mathbb{R}^{n}$, and we define $Z:=\left(z_{1}, \ldots, z_{m}\right) \in \mathbb{R}^{(n, m)}$. Then the projected eigenvalue problem

$$
K_{0} x_{m}:=P^{T} K P x_{m}=\lambda P^{T} M P x_{m}=: \lambda M_{0} x_{m}
$$

with

$$
P=K^{-1} Z\left(Z^{T} K^{-1} Z\right)^{-1} Z^{T} Z
$$

is called the condensed eigenvalue problem with general masters $z_{1}, \ldots, z_{m}$. It is easily seen that this is exactly the reduced problem introduced by Irons and Guyan if we choose $z_{1}, \ldots, z_{m}$ as unit vectors. This special case is called nodal condensation.

Since $\left(Z^{T} K^{-1} Z\right)^{-1} Z^{T} Z$ is a nonsingular matrix the condensed problem (2) is equivalent to the projection of problem (1) to the space spanned by the columns of $K^{-1} Z$. Hence, condensation is nothing else but one step of simultaneous inverse iteration with initial guess $X=M^{-1} Z \in \mathbb{R}^{(n, m)}$. Therefore, we can expect good approximation properties of condensation if we include general masters $z_{j}=M x_{j}$ where $x_{j}$ are approximate eigenvectors of problem (1) corresponding to the desired eigenvalues.

\section{Parallel condensation}

For nodal condensation the following strategy yields a coarse grained parallel algorithm developed in [4]. Suppose that the structure under consideration has been decomposed into $r$ substructures and let the masters be chosen as interface degrees of freedom. Assume that the substructures connect to each other through the master variables only. If the slave variables are numbered 
appropriately, then the stiffness matrix is given by

$$
K=\left(\begin{array}{ccccc}
K_{m m} & K_{m s 1} & K_{m s 2} & \ldots & K_{m s r} \\
K_{s m 1} & K_{s s 1} & 0 & \ldots & 0 \\
K_{s m 2} & 0 & K_{s s 2} & \ldots & 0 \\
\vdots & \vdots & \vdots & \ddots & \vdots \\
K_{s m r} & 0 & 0 & \ldots & K_{s s r}
\end{array}\right)
$$

and the mass matrix $M$ has the same block form. In this case it is easily seen that the reduced matrices $K_{0}$ and $M_{0}$ in (2) are given by

$$
K_{0}=K_{m m}-\sum_{j=1}^{r} K_{m m j}:=K_{m m}-\sum_{j=1}^{r} K_{m s j} K_{s s j}^{-1} K_{s m j}
$$

and

$$
M_{0}=M_{m m}-\sum_{j=1}^{r} M_{m m j}
$$

where

$$
M_{m m j}:=K_{m s j} K_{s s j}^{-1} M_{s m j}+M_{m s j} K_{s s j}^{-1} K_{s m j}-K_{m s j} K_{s s j}^{-1} M_{s s j} K_{s s j}^{-1} K_{s m j} .
$$

It is obvious that they can be computed completely in parallel, and that the only communication consists of one fan in process. This approach can be generalized to general masters if each of them is contained in a single substructure (cf. [2]).

In Section 1 we pointed out that good approximation properties of condensation can be expected if general masters $z_{j}=M x_{j}$ are in use where $x_{j}$ are approximate eigenvectors of problem (1). In general these will have global support, and the block structure of $K$ is destroyed. For this case we proposed a coarse grained parallelization concept the communication of which consists of two fan in processes and one broadcast to obtain the reduced matrices. Details are explained in [1].

\section{$3 \quad$ Numerical experiments}

We implemented the parallel algorithm in FORTRAN 90 using LAPACK 3 and BLAS routines for the linear algebra and MPI 1.05 for message passing. We tested the program on a heterogeneous workstation cluster consisting of one HP C3000, one HP J2240, and five HP 9000, 712/100 connected by fast-ethernet and on an HP N-Class parallel computer with 8 and $16 \mathrm{HP}$ PA $8500 / 440 \mathrm{MHz}$ processors organized as one and two clusters, respectively. 
The user can only assign each process to one of the clusters whereas the local scheduling is organized by the operating system. Moreover the computer is run in a multi-user environment. Hence we are not able to report on the load balancing of the processors.

Our test example was a finite element model of a container ship with 35262 degrees of freedom. We subdivided it into 10 substructures each of them consisting of between 1134 and 4792 unknowns, and obtained a reduced problem of dimension 2097. Besides the plain model of the ship which is called the dry model we took into account hydrodynamic masses. This model is usually called the wet model. The spectra of these two models differ quite a bit (cf. the first row of the following table which contains the relative distances). However, adding 12 dry eigenmodes corresponding to the smallest eigenvalues as general masters to the interface masters when computing the wet eigenmodes improves the eigenvalue approximations considerably (row 2 contains the relative errors of nodal condensation with interface degrees of freedom as masters only and row 3 the relative errors of condensation using 12 dry modes as general masters additionally).

\begin{tabular}{|r|r|r|r|}
\hline$\#$ & rel.dist. & nod. cond. & gen. mast. \\
\hline 1 & $3.1 \mathrm{e}-1$ & $4.9 \mathrm{e}-5$ & $4.9 \mathrm{e}-7$ \\
2 & $1.5 \mathrm{e}-1$ & $6.0 \mathrm{e}-5$ & $7.5 \mathrm{e}-7$ \\
3 & $2.7 \mathrm{e}-1$ & $2.4 \mathrm{e}-5$ & $7.9 \mathrm{e}-7$ \\
4 & $7.5 \mathrm{e}-1$ & $1.2 \mathrm{e}-4$ & $1.7 \mathrm{e}-7$ \\
5 & $1.0 \mathrm{e}+0$ & $3.9 \mathrm{e}-4$ & $3.0 \mathrm{e}-8$ \\
6 & $8.9 \mathrm{e}-1$ & $4.5 \mathrm{e}-4$ & $1.4 \mathrm{e}-7$ \\
7 & $6.0 \mathrm{e}-1$ & $6.4 \mathrm{e}-3$ & $5.6 \mathrm{e}-7$ \\
8 & $6.7 \mathrm{e}-1$ & $1.9 \mathrm{e}-2$ & $1.0 \mathrm{e}-5$ \\
9 & $3.4 \mathrm{e}-1$ & $2.1 \mathrm{e}-2$ & $1.9 \mathrm{e}-5$ \\
10 & $4.4 \mathrm{e}-1$ & $8.9 \mathrm{e}-2$ & $1.7 \mathrm{e}-4$ \\
11 & $4.7 \mathrm{e}-1$ & $1.0 \mathrm{e}-1$ & $4.2 \mathrm{e}-3$ \\
12 & $7.5 \mathrm{e}-1$ & $1.1 \mathrm{e}-1$ & $1.2 \mathrm{e}-2$ \\
\hline
\end{tabular}

Acknowledgements: Thanks are due to Christian Cabos, Germanischer Lloyd, who provided us with the finite element model of the container ship. The first author gratefully acknowledges support by the Deutsche Forschungsgemeinschaft within the Graduiertenkolleg Meerestechnische Konstruktionen. 


\section{References}

[1] B. Hofferek, A. Pelzer, and H. Voss. Global masters in parallel condensation of eigenvalue problems. Technical Report 31, Technical University of Hamburg-Harburg, Section of Mathematics, 1999. submitted to Computers \& Structures.

[2] W. Mackens and H. Voss. General masters in parallel condensation of eigenvalue problems. Parallel Computing, 25, 1999.

[3] W. Mackens and H. Voss. Nonnodal condensation of eigenvalue problems. ZAMM, 79:243 - 255, 1999.

[4] K. Rothe and H. Voss. A fully parallel condensation method for generalized eigenvalue problems on distributed memory computers. Parallel Computing, 21:907 - 921, 1995. 